\title{
Utilización del modelo de análisis psicológico del trabajo en la evaluación de rehabilitación física
}

\author{
Blanco, Gisela ${ }^{[1]}$; Luna, Silvia ${ }^{[2]}$;Quezada, Giorsari ${ }^{[3]}$
}

\section{Resumen}

El presente trabajo se planteó como objetivo, estudiar puestos de trabajo que se caracterizan por exigencias mentales, y cómo estas pueden generar efectos negativos sobre la salud del trabajador/a.

Los efectos negativos encontrados en un grupo de trabajadores/as que desempeñan como fisioterapeutas y terapeutas ocupacionales; en una institución dedicada a la rehabilitación física; fueron obtenidos a través de la aplicación de dos instrumentos. El Análisis Psicológico del Trabajo (APT) que consta de dos instrumentos específicos: análisis cualitativo y análisis orientado, permitiendo la caracterización de los puestos de trabajo y representando un algoritmo diagnostico para el análisis de los efectos. El otro instrumento utilizado es el BMS II que es un método que permite evaluar la presencia de estrés, fatiga, monotonía y desgaste profesional (burnout); los llamados efectos negativos del trabajo.

El estudio fue de tipo descriptivo, en el cual se evaluaron 16 puestos de trabajo e igual número de trabajadores (9 Fisioterapeutas y 7 Terapeutas Ocupacionales). De los 16 puestos de trabajo evaluados a través del APT; en todos, el algoritmo diagnóstico refirió que se evaluaran los efectos negativos.

Palabras claves: puestos de trabajo, exigencias mentales, efectos negativos, algoritmo diagnóstico automatizado, salud ocupacional.

\section{Abstract}

The main objective of the present study was evaluate work places which are characterized by mental demands and the negative effects they have on the health of their workers.

The negative effects found in a group of physiotherapist and occupational therapist in a institution dedicated to physical rehabilitation were obtain from the application of two instruments: The job psychological analysis (APT) which state two specific instruments: qualitative analysis and oriented analysis allowing the characterization of the work place representative algorithm diagnosis to effect analysis.

The other instrument used is the BMS II which evaluate stress, fatigue, monotony and burnout, so called the negative effects of the job.

A descriptive study which assess 16 work place in the same number of worker (9 physiotherapists and 7 occupational therapist). 16 work place evaluated using the APT the presence of negative effects were found.

Key words: work place, mental demands, negative effects, algorithm automated diagnosis, occupational health.

\footnotetext{
${ }^{[1]}$ Profesora Agregada. Escuela de Salud Pública. Facultad de Medicina. Universidad Central de Venezuela. Complejo Hospitalario José Ignacio Baldó. El Algodonal. Antemano. Caracas, Venezuela. ${ }^{[2]}$ Estudiante del último año de Terapia Ocupacional. Universidad Central de Venezuela
}

${ }^{[3]}$ Terapeuta Ocupacional. Universidad Central de Venezuela 


\section{- Introducción "}

El trabajo es, en esencia, la acción que el individuo realiza para dominar o transformar la naturaleza con el propósito de obtener la satisfacción de sus necesidades. A la vez cumple con medidas y condiciones favorables, otras desfavorables para él, lo cual se traduce en posibles factores que traen como consecuencia la presencia de riesgos para su salud, pudiendo existir la inadaptación del trabajo al hombre.

La adaptación del trabajo a la unidad socio-psico-somática que es el ser humano es uno de los postulados fundamentales en la Salud Ocupacional, que tiene como principal prioridad promover y mantener un nivel óptimo de bienestar físico, mental y social de los trabajadores. Sin embargo a partir de la Revolución Industrial caracterizada por la aplicación de las ciencias humanas al trabajo, no siempre se garantizan las condiciones adecuadas de trabajo para preservar la salud del trabajador.

Diversos autores afirman que las nuevas exigencias son cognitivas y emocionales, las cuales se presentan al trabajador durante su jornada laboral por las consecuencias de sus acciones, tanto sociales como económicas.

Con la finalidad de establecer criterios metodológicos y desarrollar una práctica para el diagnóstico y pronóstico de las relaciones hombre y actividad laboral, surge el Análisis del Trabajo. Dentro de los modelos para este análisis se encuentra, el denominado Análisis Psicológico del Trabajo(ํ) ${ }^{(1)}$. Este modelo plantea que la evaluación del trabajo debe realizarse bajo tres principios fundamentales:

1. El sistema debe aplicarse a bajo costo y con una alta sensibilidad para el diagnóstico y pronóstico de los efectos del trabajo.

2. Para lograr esta finalidad se aplica por niveles, en un primer momento se realiza una evaluación masiva denominada Análisis Cuantitativo y en un segundo momento el Análisis Orientado, culminando con el diagnóstico y pronóstico de los efectos.

3. Debe controlar la subjetividad de los/as trabajadores/as, por lo que se aplica mediante la observación de un profesional entrenado en la técnica.

Basado en este modelo Almirall y col. ${ }^{(1)}$, realizaron un procedimiento automatizado de este modelo. El tipo de trabajo que se caracteriza por exigencias principalmente psíquicas o mentales puede ser analizado a través del uso del Algoritmo Diagnóstico Automatizado; el cual permite no sólo caracterizar al puesto de trabajo sino sugiere los posibles riesgos, que a su vez pueden generar efectos negativos derivados del trabajo; tal como los describe el Modelo de Análisis Psicológico del Trabajo.

Los efectos negativos son el resultado de la carga de trabajo, expresándose generalmente cuando sobrepasan la tolerancia y reactividad del trabajador/a, como una disminución temporal de las premisas personales de rendimiento; definiendo éstas como todas las condiciones físicas y psíquicas relativamente estables que un hombre o mujer puede emplear cumpliendo tareas determinadas. Surgidas de la actividad laboral, las mismas pueden retroalimentar significativamente a la actividad y a los 
resultados del trabajo, tanto en sentido negativo como positivo, es decir, la disminución o pérdida en las premisas de rendimiento ya existentes puede reflejar una dinámica laboral desfavorable al trabajador $(\underline{2}, \underline{3})$

La carga mental de trabajo tiene su referencia concreta, en los llamados efectos negativos, que generalmente se engloban bajo los términos de fatiga, monotonía, estrés y burnout o desgaste profesional.

Esto establece la necesidad de aplicar métodos relacionados con la práctica actual de las ciencias en general y de las del trabajo en particular. Es así como en esta investigación se evaluaron las características de los puestos de trabajo de Fisioterapeutas y Terapeutas Ocupacionales que se desempeñaban en un centro de rehabilitación física, utilizando el Modelo de Análisis Psicológico del Trabajo.

Dentro de la inmensa población trabajadora, los/as trabajadores/as de la salud en general están expuestos a múltiples exigencias en sus puestos de trabajo. En primer lugar, según la naturaleza de la enfermedad de la persona que atienden, sea esta aguda o crónica, se van a observar diferentes cambios de actitud del trabajador/a, donde las enfermedades crónicas que implican el decaimiento progresivo del paciente, pueden provocar sentimientos muy arraigados de imposibilidad, frustración y desesperanzas terapéuticas.(4) ${ }^{(4)}$ tro factor que puede influir en el/la trabajador/a del área de la salud en relación al tipo de enfermedad es el temor a esta y la posibilidad de contagio( ${ }^{(4)}$.

Dentro de la amplia gama de profesionales de la salud, los del área de rehabilitación no están exentos de sufrir los efectos negativos en su puesto de trabajo, más específicamente, el Fisioterapeuta y el Terapeuta Ocupacional trabajan con el principal objetivo de mejorar la calidad de vida de múltiples pacientes con discapacidades físicas o mentales, personas que debido a su condición sufren una serie de cambios negativos en su vida y ante esto lo que se pretende lograr es la adaptación del individuo al contexto de su nueva realidad.

Algunas investigaciones realizadas señalan que estos profesionales, trabajan de forma intensa, íntima y continua con personas con problemas físicos, mentales, emocionales y sociales, generando tensión asociada con el proceso de tratamiento y enseñanza al paciente, facilitando el riesgo de desarrollar desgaste profesional. ${ }^{(5)}$

Una característica común de las labores pertinentes a estos profesionales de la salud, es la responsabilidad ante la toma de decisiones que afectan directamente de manera positiva o negativa el bienestar de otro, sumado a esto la confianza que suele depositar el paciente y su familia en el personal de salud son algunos de los estresores que se han encontrado en el ejercicio de estos roles. ${ }^{(5)}$

En cuanto a la naturaleza y relación con el paciente, el personal asistencial en rehabilitación, suele enfrentar las diferentes reacciones y cambios de actitud que tienen los pacientes, familiares y seres queridos que podrían variar desde sentimientos confusos, enfado exagerado, dependencia o necesidad de mantener un control excesivo, de igual manera el personal aporta a esta relación un cúmulo de importantes experiencias con otros pacientes que inconscientemente influyen en la manera de acercarse a otros afectando la retroalimentación esperada. 
Por las razones antes expuestas se ha considerado y se considera importante evaluar puestos de trabajo de los profesionales que se desenvuelven dentro del sector salud, específicamente en el área de Rehabilitación, para ello es necesario la participación de un profesional capacitado que evalué los efectos negativos que afectan a los trabajadores de esta área .

Como se mencionó con anterioridad, El Análisis Psicológico del Trabajo es un método donde se determinan las tareas que impone un trabajo y las habilidades, conocimientos, especialidades y responsabilidades requeridas del trabajo para su adecuado ejercicio y que diferencian al trabajador de todos los demás y a su vez diagnostica, pronostica, promociona y previene aquellas condiciones del ambiente laboral a las cuales está expuesto el trabajador. ${ }^{(1)}$

El Análisis Psicológico del Trabajo es un método que no requiere ser aplicado solo por psicólogos pero sí por un personal conocedor de la actividad laboral y entrenado en el uso de instrumentos de esta naturaleza, que pueden considerar las condiciones que producen alteraciones a la salud, en particular las exigencias psíquicas de la tarea.

Son diversos los profesionales implicados en la valoración de puestos de trabajo, al igual que múltiples son los métodos para esta evaluación. Dentro de esta interdisciplinaridad se encuentra el Terapeuta Ocupacional; el cual cumple un papel fundamental en el análisis ocupacional del individuo, analizando y promoviendo el mejoramiento de las condiciones del trabajo en función de las necesidades y capacidades del hombre a nivel psíquico, físico, social y emocional.

\section{- Metodología}

El tipo de estudio utilizado en esta investigación fue descriptivo, teniendo como principal objetivo medir una serie de características descritas por el Análisis Psicológico del Trabajo; en un conjunto de puestos de trabajo de Fisioterapeuta y Terapeuta Ocupacional.

En esta investigación se seleccionaron un conjunto de puestos de trabajo del área de la rehabilitación física, llevando a cabo una previa evaluación de las tareas específicas de cada uno de los puestos, mediante la aplicación de procedimientos de evaluación como el Análisis Psicológico del Trabajo; es importante señalar que el APT es uno de los diversos modelos de análisis del trabajo. Una vez obtenidos los resultados, se procedió a analizar y verificar si era necesario continuar con las recomendaciones referidas por el Algoritmo Diagnóstico, para lograr una mayor precisión del objeto en estudio. Uno de los métodos de evaluación de efectos negativos es el BMS II (Belasting Monotonie Saturation), el cual señalará la presencia de estos efectos que podrían interferir en la ejecución de las tareas de los puestos en estudio.

La población estudiada fue de 9 Fisioterapeutas y 7 Terapeutas Ocupacionales en ambos turnos; turno de la mañana 7:00am a 1:00pm y turno de la tarde 1:00pm a 7:00pm; con un total de 16 trabajadores. De este grupo de trabajadores/as 9 son de sexo femenino y 7 masculino con una edad promedio de 34.6 años donde el límite menor es de 30 años y el límite mayor es de 47 años. En cuanto a los años de experiencia de los trabajadores varía entre 6 años y 26 años de experiencia, con un promedio de 13.75 años, de los cuales, 12 trabajadores ocupan un cargo asistencial y 
4 ocupan el cargo de coordinador o supervisor y también ejercen funciones asistenciales.

Los instrumentos empleados fueron el Análisis Psicológico del Trabajo (APT), un procedimiento automatizado para el diagnóstico de los efectos negativos del trabajo, el cual puede ser aplicado en un puesto particular, en una empresa o en cualquier sector de la economía. Este procedimiento se lleva a cabo a través de la observación directa del puesto de trabajo a evaluar.

El APT consta de dos instrumentos de evaluación:

El análisis cualitativo (AC): se realiza generalmente utilizando el método de la observación sistémica de la actividad a evaluar, completando la información con el juicio de tantos expertos como el investigador considere necesario.

El análisis orientado (AO): consta de una lista estandarizada, que cuenta con 99 características a evaluar, representando los atributos generales del trabajo. El AO presenta sus resultados en un gráfico cuanti-cualitativo de fácil interpretación, el cual considera grados de tolerancia y adaptación del trabajador ante su labor.

Si los resultados arrojados por análisis automatizado señalaba que la evaluación de efectos negativos podía incidir en la salud del trabajador, se utilizaría la prueba denominada Belasting Monotonie Saturation (BMS II) (Richter y Plath, 1984) ${ }^{[5]}$; la cual evalúa la presencia de los cuatro efectos negativos descritos por estos autores: Fatiga, Monotonía, Estrés y Burnout.

Este instrumento consta de dos escalas paralelas A y B cada una cuenta con 40 items y sus autores recomiendan aplicar la primera a los 30 minutos de iniciado el turno de trabajo (por ejemplo la forma A), y luego el siguiente, 30 minutos antes de culminar la jornada laboral (la forma $B$ ).

Los resultados se dan en términos de tres tipos de escalas:

Escala 1: Buen estado de salud.

Escala 2: Perjuicios lógicos del estado de salud, se deben realizar investigaciones profundas (análisis del contenido del trabajo, nivel de activación psicofisiológica, entre otros) para realizar medidas de organización.

Escala 3: Fuertes daños en el estado de salud; es indispensable tomar medidas para la estructuración del trabajo y de la organización.

En cuanto al procedimiento para la aplicación del APT lo realizaron evaluadores (terapeutas ocupacionales), con previo conocimiento de la actividad laboral y entrenados en el uso de los sistemas. Una vez seleccionados los puestos de trabajo y aplicado el APT, se realizó una segunda fase a través de la aplicación del BMS II, efectivamente en los resultados obtenidos a través del Análisis Psicológico del Trabajo se señalan condiciones desfavorables en el trabajador, se procede a aplicar el BMSII (A y B). Los Fisioterapeutas y Terapeutas Ocupacionales respondieron el cuestionario, siguiendo las instrucciones que este contiene. Tomando en cuenta que uno de ellos se aplicó media hora después de iniciada la jornada y el otro media hora antes de culminarla. 


\section{- Resultados}

En todos los puestos evaluados a través del Análisis Psicológico del Trabajo, el Algoritmo Diagnóstico Automatizado refirió la evaluación de efectos negativos. Los resultados del APT se expresan en cuatro categorías de análisis de puestos como son: contenido extenso, contenido restringido, condiciones adversas de trabajo y exigencias especiales para desempeñar las tareas. En función de ello, estos puestos de trabajo presentan características especiales, dentro de las cuales la más significativa es referente a la intensidad del trabajo dado por la existencia de contenido extenso y restringido de las tareas; es decir gran cantidad de trabajo.

De los 16 puestos referidos por el Algoritmo Diagnóstico para la evaluación de efectos negativos, por la presencia de condiciones adversas y contenido extenso de trabajo; se encontró que al aplicar el instrumento BMSII que evalúa los cuatro efectos negativos (fatiga, monotonía, estrés y desgaste profesional), ocho de ellos presentaron efectos negativos. En los Puestos de los Fisioterapeutas del Turno de la mañana; el sujeto 2 presentó estrés y fatiga antes de iniciar la jornada laboral, el sujeto 3 fatiga también antes de iniciar la jornada laboral, el sujeto 5 estrés y fatiga antes de la jornada y monotonía una vez culminada la jornada laboral. En este turno llama la atención que los trabajadores que presentaron efectos negativos los refirieron solo antes de iniciar la jornada laboral. En el turno de la tarde el sujeto 7 presenta estrés antes de iniciar el turno y fatiga al finalizar.

En los puestos de Terapeutas Ocupacionales del turno de la mañana se encontró fatiga en el sujeto 10 antes de iniciar la jornada y en el sujeto 13 antes y después. En el turno de la tarde el sujeto 15 presentó estrés antes de la jornada laboral y fatiga antes y después; y finalmente el sujeto 16 presenta fatiga luego de culminar la jornada laboral. (Ver tablas 1 y 2 ).

Al comparar ambos turnos de trabajo no se encontraron diferencias significativas al igual que en relación al tipo de cargo: Fisioterapeutas y Terapeutas Ocupacionales.

Tabla 1. Resultados del BMSII en Fisioterapeutas en el Turno de la Mañana

\begin{tabular}{|c|c|c|c|c|c|c|c|c|c|}
\hline \multirow{3}{*}{\begin{tabular}{|l|} 
Turno \\
AM \\
$7: 00$ a \\
$12: 00$ \\
\end{tabular}} & & \multicolumn{2}{|l|}{ Estrés } & \multicolumn{2}{|l|}{ Fatiga } & \multicolumn{2}{|c|}{\begin{tabular}{|l|} 
Desgaste \\
Profesional
\end{tabular}} & \multicolumn{2}{|c|}{ Monotonía } \\
\hline & & Antes & Después & Antes & Después & Antes & Después & Antes & Después \\
\hline & Sujeto 1 & 0,59 & 0,59 & 0,55 & 0,60 & 0,58 & 0,60 & 0,61 & 0,63 \\
\hline & Sujeto 2 & 0,48 & 0,52 & 0,48 & 0,52 & 0,57 & 0,59 & 0,59 & 0,52 \\
\hline & Sujeto 3 & 0,59 & 0,57 & 0,47 & 0,60 & 0,57 & 0,60 & 0,56 & 0,60 \\
\hline & Sujeto 4 & 0,55 & 0,57 & 0,53 & 0,56 & 0,52 & 0,60 & 0,59 & 0,59 \\
\hline & Sujeto 5 & 0,47 & 0,55 & 0,49 & 0,53 & 0,54 & 0,56 & 0,59 & 0,47 \\
\hline & Sujeto 6 & 0,55 & 0,56 & 0,52 & 0,56 & 0,57 & 0,61 & 0,61 & 0,60 \\
\hline
\end{tabular}


Tabla 2. Resultados del BMSII en Fisioterapeutas en el Turno de la Tarde

\begin{tabular}{|c|c|c|c|c|c|c|c|c|c|}
\hline \multirow{3}{*}{\begin{tabular}{|l|} 
Turno \\
PM \\
1:00 a \\
7:00 \\
\end{tabular}} & & \multicolumn{2}{|l|}{ Estrés } & \multicolumn{2}{|l|}{ Fatiga } & \multicolumn{2}{|c|}{\begin{tabular}{|l|} 
Desgaste \\
Profesional
\end{tabular}} & \multicolumn{2}{|c|}{ Monotonía } \\
\hline & & Antes & Después & Antes & Después & Antes & Después & Antes & Después \\
\hline & Sujeto 7 & 0,49 & 0,56 & 0,55 & 0,49 & 0,55 & 0,59 & 0,59 & 0,51 \\
\hline & Sujeto 8 & 0,62 & 0,55 & 0,53 & 0,56 & 0,60 & 0,58 & 0,55 & 0,62 \\
\hline & Sujeto 9 & 0,58 & 0,55 & 0,62 & 0,53 & 0,60 & 0,58 & 0,64 & 0,56 \\
\hline
\end{tabular}

Tabla 3. Resultados del BMSII en Terapeutas Ocupacionales en el Turno de la mañana

\begin{tabular}{|c|c|c|c|c|c|c|c|c|c|}
\hline \multirow{3}{*}{\begin{tabular}{|l|} 
Turno \\
AM \\
$7: 00$ a \\
$12: 00$ \\
\end{tabular}} & & \multicolumn{2}{|l|}{ Estrés } & \multicolumn{2}{|l|}{ Fatiga } & \multicolumn{2}{|c|}{\begin{tabular}{|l} 
Desgaste \\
Profesional
\end{tabular}} & \multicolumn{2}{|c|}{ Monotonía } \\
\hline & & Antes & Después & Antes & Después & Antes & Después & Antes & Después \\
\hline & $\begin{array}{l}\text { Sujeto } \\
10\end{array}$ & 0,52 & 0,59 & 0,49 & 0,56 & 0,53 & 0,62 & 0,59 & 0,50 \\
\hline & $\begin{array}{l}\text { Sujeto } \\
11\end{array}$ & 0,50 & 0,57 & 0,57 & 0,55 & 0,57 & 0,60 & 0,62 & 0,60 \\
\hline & $\begin{array}{l}\text { Sujeto } \\
12\end{array}$ & 0,54 & 0,59 & 0,57 & 0,56 & 0,57 & 0,65 & 0,62 & 0,57 \\
\hline & \begin{tabular}{|l|} 
Sujeto \\
13
\end{tabular} & 0,57 & 0,57 & 0,46 & 0,41 & 0,53 & 0,58 & 0,61 & 0,54 \\
\hline
\end{tabular}

Tabla 4. Resultados del BMSII en Terapeutas Ocupacionales en el Turno de la Tarde

\begin{tabular}{|c|c|c|c|c|c|c|c|c|c|}
\hline \multirow{3}{*}{\begin{tabular}{|l} 
Turno \\
PM \\
$1: 00$ a \\
$7: 00$
\end{tabular}} & \multirow[b]{3}{*}{$\begin{array}{l}\text { Sujeto } \\
14\end{array}$} & \multicolumn{2}{|l|}{ Estrés } & \multicolumn{2}{|l|}{ Fatiga } & \multicolumn{2}{|c|}{\begin{tabular}{|l} 
Desgaste \\
Profesional
\end{tabular}} & \multicolumn{2}{|c|}{ Monotonía } \\
\hline & & \multirow{2}{*}{\begin{tabular}{|l|} 
Antes \\
0,58 \\
\end{tabular}} & Después & \multirow{2}{*}{\begin{tabular}{|l|} 
Antes \\
0,54
\end{tabular}} & Después & \multirow{2}{*}{\begin{tabular}{|l|} 
Antes \\
0,63
\end{tabular}} & \multirow{2}{*}{\begin{tabular}{|l|} 
Después \\
0,58
\end{tabular}} & \multirow{2}{*}{\begin{tabular}{|l|} 
Antes \\
0,61
\end{tabular}} & \multirow{2}{*}{\begin{tabular}{|l|} 
Después \\
0,62
\end{tabular}} \\
\hline & & & 0,54 & & 0,57 & & & & \\
\hline & \begin{tabular}{|l} 
Sujeto \\
15
\end{tabular} & 0,47 & 0,57 & 0,47 & 0,49 & 0,57 & 0,63 & 0,51 & 0,64 \\
\hline & $\begin{array}{l}\text { Sujeto } \\
16\end{array}$ & 0,58 & 0,54 & 0,50 & 0,42 & 0,60 & 0,63 & 0,63 & 0,59 \\
\hline
\end{tabular}

\section{- Discusión "}

El Análisis Psicológico del Trabajo, permitió realizar una evaluación de los puestos de trabajo a muy bajo costo y de forma eficaz y rápida. En todos los puestos evaluados se pudo apreciar que las condiciones en que se realizan las tareas (condiciones adversas, contenido extenso del trabajo, etc) muy probablemente condicionaron la recomendación por parte del Algoritmo Diagnóstico de sugerir la evaluación de efectos negativos del trabajo.

La versatilidad del Análisis Psicológico del Trabajo (APT) lo hace un instrumento que se adapta a la evaluación de los puestos de trabajo con exigencias mentales o 
cognitivas principalmente, siendo su aplicación de bajo costo, rápida y óptima. Paralelamente, a través de esta evaluación se pueden predecir algunos de los efectos negativos del trabajo (fatiga, monotonía, estrés y desgaste profesional o burnout) constituyendo una herramienta valiosa en la esfera de la prevención de posibles problemas y enfermedades en los/as trabajadores/as.

Por otra parte los resultados obtenidos en cuanto a la presencia de efectos negativos del trabajo permitieron apreciar que la mitad de los trabajadores evaluados, presentaron algún efecto negativo del trabajo como resultado en buena medida del tipo de actividad laboral que realizan, sin dejar a un lado la influencia de las variables extralaborales.

La presencia de efectos negativos como estrés y desgaste profesional ha sido señalado por diversos autores. Robers y Dodson ${ }^{(5)}$ refieren que existe una relación directa entre la presencia de desgaste profesional y la cantidad de pacientes a atender, es decir, mientras mas población a tratar tiene un terapeuta ocupacional más serán las probabilidades de sufrir desgaste profesional (burnout); también influyen el número de horas de contacto con el paciente, la edad del terapeuta y los años de experiencia. El estudio señala que la correlación es directa para todas las variables mencionadas.

Uno de las aspectos mas frecuentes dentro del cuadro de los terapeutas ocupacionales es el agotamiento emocional; igualmente se afirma que las características de la personalidad, la salud física con que cuenta el trabajador, las relaciones familiares y las consecuencias orgánicas se relacionan con la presencia y severidad del síndrome; por ejemplo, las características de la personalidad son asociadas con el comportamiento emocional, la despersonalización y falta de sentimiento del logro. La salud física y las relaciones familiares se asocian con el agotamiento emocional.

Se señala, que el miedo a la negligencia es uno de los causas de tensión que ha recibido recientemente más atención por parte de los profesionales de la medicina, también muchos estudios han identificado la carga excesiva de trabajo como una fuente importante de desgaste profesional, específicamente con el síntoma de agotamiento emocional, siendo este factor de riesgo indicativo que guarda estrecha relación con las dimensiones del desgaste profesional. $(\underline{6})$

Merece especial atención la recomendación de lograr ambientes de trabajo que ofrezcan actividades con pequeños grupos de pacientes, la necesidad de trabajar en equipo y contar con el apoyo de supervisores y colegas, donde los terapeutas ocupacionales cuenten con una independencia suficiente que le permita dar significado a su trabajo; todo esto permitirá la creación de un ambiente laboral favorable al bienestar del terapeuta ocupacional. ${ }^{(\underline{7})}$

Por su parte los fisioterapeutas también atienden a personas con las mismas discapacidades que los terapeutas ocupacionales, teniendo contacto con pacientes que tienen rasgos de depresión, angustia, ansiedad y temor por su enfermedad y discapacidad factores que pueden desencadenar el desgaste profesional en estos trabajadores. La mayoría de los fisioterapeutas experimentan cambios bruscos en su ambiente de trabajo, experimentando tensión, descontento, pérdida de control sobre su trabajo, sin embargo también se demostró de la mayoría lograba adaptarse efectivamente a estos cambios. $\underline{(ㄱ)}$ 
Por último y no por ello menos importante, la capacitación de Terapeutas Ocupacionales en el manejo de diferentes procedimientos de análisis del trabajo, entre ellos el Análisis Psicológico del Trabajo, es fundamental; fortaleciendo su rol como miembro del equipo de salud, y más específicamente como miembro del equipo interdisciplinario de la Salud Ocupacional.

\section{- Referencias}

(1) Almirall Pedro. Ergonomía Cognitiva: Apuntes para su Aplicación en Salud y Trabajo: Imprenta Universitaria de la Universidad Central de Venezuela., 2001; 26-38.

(2) Delgado J. Ergonomía Cognitiva. Departamento de Psicología Experimental y Fisiología del Comportamiento de la Universidad de Granada [serial online] [citado 14 Noviembre 2004];1(1). Disponible en: http://www.psicología-online.com/artículos/2004/ergonomía.shtml

(3) Welford, A.T. "La Carga de Trabajo Mental como una función de la demanda, la capacidad y la estrategia del operador" Ergonomics. 1978; 21: 151-158.

(4) Gutiérrez J. Estrés en los trabajadores de la Salud. CEPIS Centro Panamericano de Ingeniería Sanitaria y Ciencias del Ambiente [serial online] [citado 06 Noviembre 2004]; 8(8). Disponible en: http://www.cepis.ops-oms.org/

(5) Rogers J, Dodson S. Burnout in Occupational Therapists. The American Journal of Occupational Therapy.1987. 28: 787-792

(6) González J, Moreno R, Peñacoba C, et al. Burnout in Occupational Therapy: an analisys focused on the level of individual and organizational consequences. Psychology in Spain, 2004 Vol. 8: 98-105

(7) Collins, J. Un Método para la evaluación de los efectos de la carga psíquica Tesis de Maestría en Salud Pública. Facultad de Salud. Ministerio de Salud Pública. La Habana, 1991; 89-96 